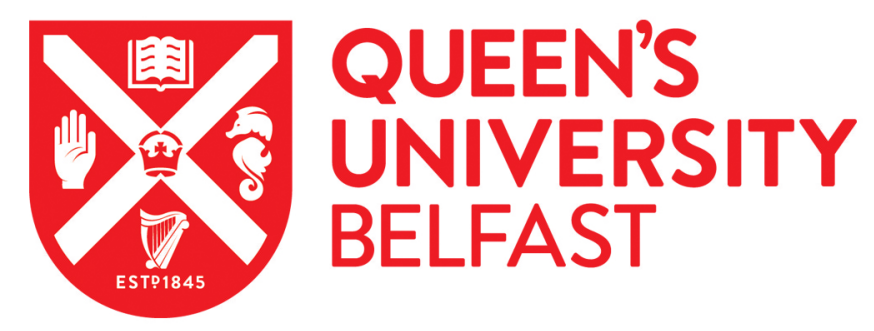

\title{
Topology of megagauss magnetic fields and of heat-carrying electrons produced in a high-power laser-solid interaction
}

Lancia, L., Albertazzi, B., Boniface, C., Grisollet, A., Riquier, R., Chaland, F., Le Thanh, K. C., Mellor, P., Antici, P., Buffechoux, S., Chen, S. N., Doria, D., Nakatsutsumi, M., Peth, C., Swantusch, M., Stardubtsev, M.,

Palumbo, L., Borghesi, M., Willi, O., ... Fuchs, J. (2014). Topology of megagauss magnetic fields and of heatcarrying electrons produced in a high-power laser-solid interaction. Physical Review Letters, 113(23), [235001]. https://doi.org/10.1103/PhysRevLett.113.235001

Published in:

Physical Review Letters

Document Version:

Peer reviewed version

Queen's University Belfast - Research Portal:

Link to publication record in Queen's University Belfast Research Portal

Publisher rights

Copyright 2014 American Physical Society

General rights

Copyright for the publications made accessible via the Queen's University Belfast Research Portal is retained by the author(s) and / or other copyright owners and it is a condition of accessing these publications that users recognise and abide by the legal requirements associated with these rights.

Take down policy

The Research Portal is Queen's institutional repository that provides access to Queen's research output. Every effort has been made to ensure that content in the Research Portal does not infringe any person's rights, or applicable UK laws. If you discover content in the Research Portal that you believe breaches copyright or violates any law, please contact openaccess@qub.ac.uk. 


\title{
Topology of Megagauss Magnetic Fields and of Heat-Carrying Electrons Produced in a High-Power Laser-Solid Interaction
}

\author{
L. Lancia, ${ }^{1,2}$ B. Albertazzi, ${ }^{2,3}$ C. Boniface, ${ }^{4,}$ A. Grisollet, ${ }^{4}$ R. Riquier, ${ }^{2,4}$ F. Chaland, ${ }^{4}$ K.-C. Le Thanh, ${ }^{4}$ Ph. Mellor, ${ }^{4}$ \\ P. Antici, ${ }^{1}$ S. Buffechoux, ${ }^{2}$ S. N. Chen, ${ }^{2}$ D. Doria, ${ }^{5}$ M. Nakatsutsumi, ${ }^{2}$ C. Peth, ${ }^{6}$ M. Swantusch, ${ }^{6}$ M. Stardubtsev, ${ }^{7}$ \\ L. Palumbo, ${ }^{1}$ M. Borghesi, ${ }^{5}$ O. Willi, ${ }^{6}$ H. Pépin, ${ }^{3}$ and J. Fuchs ${ }^{2,7, \dagger}$ \\ ${ }^{1}$ Dipartimento SBAI, Università di Roma La Sapienza, Via Antonio. Scarpa 14, 00161 Rome, Italy \\ ${ }^{2}$ LULI, École Polytechnique, CNRS, CEA, UPMC, 91128 Palaiseau, France \\ ${ }^{3}$ INRS-EMT, Varennes, Québec J3X 1S2, Canada \\ ${ }^{4}$ CEA, DAM, DIF, F-91297 Arpajon, France \\ ${ }^{5}$ School of Mathematics and Physics, The Queen's University of Belfast, Belfast BT7 1NN, United Kingdom \\ ${ }^{6}$ Heinrich Heine Universität Düsseldorf, D-40225 Düsseldorf, Germany \\ ${ }^{7}$ Institute of Applied Physics, 46 Ulyanov Street, 603950 Nizhny Novgorod, Russia
}

(Received 3 March 2014; published 2 December 2014)

\begin{abstract}
The intricate spatial and energy distribution of magnetic fields, self-generated during high power laser irradiation (at $I \lambda^{2} \sim 10^{13}-10^{14} \mathrm{~W} \cdot \mathrm{cm}^{-2} \cdot \mu \mathrm{m}^{2}$ ) of a solid target, and of the heat-carrying electron currents, is studied in inertial confinement fusion (ICF) relevant conditions. This is done by comparing proton radiography measurements of the fields to an improved magnetohydrodynamic description that fully takes into account the nonlocality of the heat transport. We show that, in these conditions, magnetic fields are rapidly advected radially along the target surface and compressed over long time scales into the dense parts of the target. As a consequence, the electrons are weakly magnetized in most parts of the plasma flow, and we observe a reemergence of nonlocality which is a crucial effect for a correct description of the energetics of ICF experiments.
\end{abstract}

DOI: 10.1103/PhysRevLett.113.235001

PACS numbers: 52.25.Fi, 52.38.Fz, 52.65.Kj, 52.70.Nc

Characterizing magnetic $(B)$ fields in plasmas is a subject of major interest since $B$ fields influence many plasma phenomena such as heat transport or hydrodynamic instability formation. In laser-produced plasmas, strong (>1 MG) magnetic fields can be self-generated by the noncollinear electron density and temperature gradients [1]. Their study is important for inertial confinement fusion (ICF), but also for astrophysical phenomena such as magnetic reconnection $[2,3]$ since the initial topology of laserdriven $B$ fields determines their reconnection dynamics $[4,5]$.

The study of $B$ fields in plasmas is intrinsically connected to that of electron heat transport, which in laser plasmas is commonly nonlocal, as the linearized Spitzer-Härm theory [6] breaks down due to the long mean free path of high velocity electrons compared to the temperature gradient scale length. Such an intricate connection is manifested in several ways: nonclassical heat flow modifies the speed of the Nernst advection, which results in $B$-field convection and compression by the heat flow down a temperature gradient, i.e., towards denser and colder regions [7], and hence, influences the $B$-field evolution [8,9]. In turn, $B$ fields act back on electron transport by reducing the mobility of heat-carrying electrons. Moreover, through the Righi-Leduc heat flow [8], $B$ fields act on the electrons in the direction orthogonal to both the $B$ field and to the electron temperature gradient, causing a bending of the heat flux [10]. From an ICF point of view, accurate modeling of both quantities is important to optimize both the implosion symmetry of direct-drive capsules, and the $\mathrm{x}$-ray emission of high- $Z$ materials [11] inside indirect-drive ICF cavities.

The need for characterizing the $B$-field-heat-flow coupling has led to a number of studies using the available modeling tools, i.e., kinetic or radiation-hydrodynamics codes. Achieving a quantitative modeling capability of ICF-relevant experiments has, however, been elusive up to now, due to the conflicting pictures arising from those studies. Indeed, so far, hydrodynamic models, able to model nanosecond time scales, exhibit slowly evolving magnetic fields that are convected to low-density regions [12-15]. We should note, here, that these models use either some flux-limited [16] or nonlocal transport model [17], but are restricted (see, e.g., [16]) to the classical (Braginskii) [18] description of the Nernst advection. When quantitatively compared to experiments, they were found to underestimate the $B$ fields radial advection $[17,19]$. Conversely, kinetic descriptions, however limited to short time scales (tens of picoseconds), exhibit rapidly advected fields compressed into the dense parts of the target. Such topology of $B$ fields was found to be consistent with measurements [20], although these were performed at higher intensities, i.e., in a regime where hot electrons become dominant and could significantly affect thermal conduction.

To resolve these issues and progress toward quantitative modeling in ICF-relevant conditions, we worked on an 
improved magnetohydrodynamic (MHD) description [21] that, by fully taking into account the nonlocal nature of the transport, can overcome the standard limitations of the classical (Braginskii) [18] model of magnetized transport. This description is benchmarked against proton radiography measurements of $B$ fields performed in various conditions, on two different laser facilities (LULI2000 and TITAN-JLF). With a quantitative agreement between the measurements and the improved modeling, we observe that $B$ fields are distributed in a topology that significantly differs from the picture derived from previous hydrodynamic modeling: they are rapidly advected radially and compressed into the dense parts of the target, even over long (ns) time scales. Based on simulation we performed at an energy level of $10 \mathrm{~kJ}$, corresponding to one quadruplet of the National Ignition Facility (NIF) and Laser Megajoule (LMJ) facility, we verify that this $B$-field topology scales up to full ICF conditions. By further simulating full ICF experiments with our improved modeling, we also observe that this significantly affects the prediction of the plasma evolution of full ICF experiments compared to the standard (flux-limited) approach [21]. The quantitative agreement between measurements and MHD code predictions further demonstrates that simulations over hydrodynamic, i.e., long-time scales, can be achieved, overcoming the common limitations of the more accurate kinetic (e.g., FokkerPlanck) tools.

First, let us summarize the typical distribution of $B$ fields observed in the experiments. As shown in Fig. 1(a), by sending a beam of broadband probing protons [22], we can retrieve information on the $B$-field topology [23] through the deflections impaired on the probing protons by the $B$ fields they cross. In this experimental arrangement, the detection is performed by a stack of films [see Fig. 1(a)] where each film detects a narrow energy range of protons; thus, the deflected pattern induced by the $B$ fields in a given film corresponds to an integration time of less than ten picoseconds. Figure 1(b) shows an image of such a proton beam, here obtained at LULI2000, after it has passed through a $25 \mu \mathrm{m}$ Mylar target, following the irradiation by a $I \lambda_{0}^{2} \simeq 3 \times 10^{13} \mathrm{~W} . \mathrm{cm}^{-2} . \mu \mathrm{m}^{2}$ intense laser pulse $(200 \mathrm{~J}$ incident on target, $100 \mu \mathrm{m}$ FWHM, $4 \mathrm{~ns}$, of wavelength $\lambda_{o}=532 \mathrm{~nm}$ ) [17]. The field structure is, here, probed by the protons $600 \mathrm{ps}$ after the beginning of the main beam irradiation (with time zero corresponding to the foot of the nanosecond pulse that has a $1 \mathrm{~ns}$ linear rise, followed by a 3 ns plateau and a $200 \mathrm{ps}$ linear decrease). The spatial resolution of the probing, given by the proton beam source size [24] is less than ten microns.

Hydrodynamics as well as kinetic simulations suggest that the induced $B$ fields develop mostly on the target surface, are azimuthal about the laser axis $(z)$, and polarized clockwise looking along the laser direction. In the arrangement shown in Fig. 1(a), the probing protons are, therefore, swept out from the central regions where the $B$ field

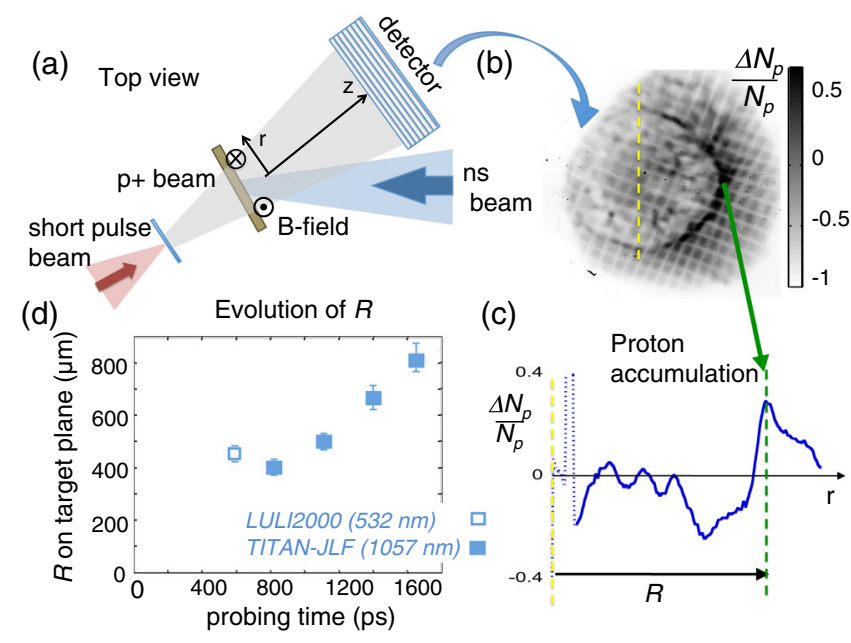

FIG. 1 (color online). (a) Proton probing of $B$ fields: experimental setup. (b) Typical image of the dose modulation (see scale on the right) in the proton beam, as recorded on the film detector. The regular pattern superimposed on the probing proton beam and observed on the image is due to the presence of a mesh located between the proton source and the probed target. (c) Radial lineout of (b) angularly averaged. The arrow points to the position $R$ of the deflected and accumulated protons with respect to the laser axis. (d) Temporal evolution of $R$ [see (c) and text].

extends. Hence, this will induce a lower dose (whiter zone on the image) of protons inside the $B$-field zone and an accumulation of protons (darker ring on the image) at the edge of the $B$-field zone. This corresponds to what is seen in Fig. 1(b).

The proton dose accumulation exhibits a toroidal shape that reproduces the azimuthal geometry of the field distribution. As shown in Fig. 1(c), the radius $(R)$ of the darker ring [arrow in Fig. 1(c)] quantifies the maximum radial extent of the fields projected on the target plane; particular importance is given to this parameter since it provides a measure of the field advection velocity. Exactly the same deflection pattern displayed in Fig. 1(b) is again observed on experiments conducted at TITAN JLF using similar geometry but different laser beam parameters in order to test our modeling in other conditions: $360-420 \mathrm{~J}$ incident on target, $150 \mu \mathrm{m}$ FWHM, 2 ns duration, wavelength $\lambda_{o}=1057 \mathrm{~nm}$, and a $I \lambda_{o}^{2} \simeq 10^{14} \mathrm{~W} \cdot \mathrm{cm}^{-2} \cdot \mu \mathrm{m}^{2}$ irradiating the same Mylar target. In this second experiment, the field evolution is probed at different time steps. The measured values of $R$ as probed at different times and for the two experiments are summarized in Fig. 1(d).

To model the experiments, we now test an improved version of the 2D hydroradiative code FCI2 developed at CEA [21]. FCI2 is a 2D, arbitrary Lagrangian-Eulerian, two-temperature code [25] that can use, as most codes, a flux limited Spitzer-Harm or Braginskii transport model, but also a two-dimensional kinetic model for nonlocal transport including the effect of $B$ fields through a resistive MHD package [26]. We found that, in order to reach a 
quantitative agreement with the experimental data of Fig. 1, the code had to be critically improved in the coupled description of electron transport and $B$-field growth and evolution. This coupling appears through the $B$-field evolution equation. This is obtained by substituting the electron momentum equation into Faraday's equation. Thus, the induction equation takes the form

$$
\begin{aligned}
\frac{\partial \mathbf{B}}{\partial t}= & \nabla \times(\mathbf{U} \times \mathbf{B})-\nabla \times\left(\frac{1}{\sigma \mu_{0}} \nabla \times \mathbf{B}\right) \\
& +\frac{\nabla T_{e} \times \nabla n_{e}}{e n_{e}}-\nabla \times\left(\frac{\mathbf{J} \times \mathbf{B}}{e n_{e}}\right) .
\end{aligned}
$$

Here, $\mathbf{U}=\mathbf{V}_{\text {Flow }}+\mathbf{V}_{\text {Nernst }}$ is the advection velocity, given by the contribution of magnetic convection by the plasma flow velocity $\mathbf{V}_{\text {Flow }}$ and by the so-called Nernst velocity $\mathbf{V}_{\text {Nernst }} ; \sigma$ is the conductivity, $T_{e}$ the electron temperature, $e$ the unit charge, $n_{e}$ the electron density, and $\mathbf{J}$ the electron current density.

The first and second terms in Eq. (1) represent the convection and diffusion of the magnetic field. The third term is the Biermann-Battery source field $\left[\sim \nabla \times\left(\mathbf{P}_{e} / e n_{e}\right)\right.$ where $\mathbf{P}_{e}$ is the electron pressure], arising from the nonparallel $\nabla T_{e}$ and $\nabla n_{e}$ [27], and the last term is the Hall effect source. We are, here, in a regime where the ratio between thermal and magnetic pressure, $\beta \sim\left|\left(\mathbf{P}_{e} / e n_{e} L\right)\right| /\left|\left(\mathbf{B}^{2} / e n_{e} L\right)\right|$, where $L$ is the characteristic magnetic field length, is $\gg 1$. Hence, the Hall effect is negligible at first order.

In these high- $\beta$ and high- $R_{m} \quad\left(R_{m} \gg 1\right.$, where the Reynolds magnetic number $R_{m}$ is the ratio between $B$-field advection and diffusion in the plasma) conditions, the magnetic field is mainly convected by the plasma. The expression of the Nernst velocity $\mathbf{V}_{\text {Nernst }}$ as treated in fluid simulations reads $\mathbf{V}_{\text {Nernst }}=\mathbf{Q}_{\mathrm{NL}} /\left((1 / \gamma-1) P_{e}\right)$, where $\mathbf{Q}_{\mathrm{NL}}$ is the electron heat flux written in a nonlocal formalism [26,28], and $\gamma$ the adiabatic index (we used $\gamma=5 / 3$ here)

We stress that a key point here is to treat, fully and consistently, the nonlocal nature of the heat transport through the $\mathbf{Q}_{\mathrm{NL}}$ term, in order to overcome the limitation imposed by the classical Braginskii model. This latter model (see, e.g., [16]) implies a local approach in modeling heat transport, and hence, hinders any real possibility of quantitative correct modeling. Finally, a thermomagnetic effect, the Righi-Leduc effect [8], is also included in fluid simulations.

To validate our improved hydrodynamic approach, we first compared simulations performed using a kinetic model to FCI2 simulations for a range of laser intensities, density profiles, and target materials [using both low $Z$ and high $Z$ $(\mathrm{Au})]$ and found the two to be in agreement over the first $\sim 100$ ps of the interaction [21]. To validate our model over longer time scales, we compared the simulation results to the experimental ones of Fig. 1. For this, FCI2 is run in two
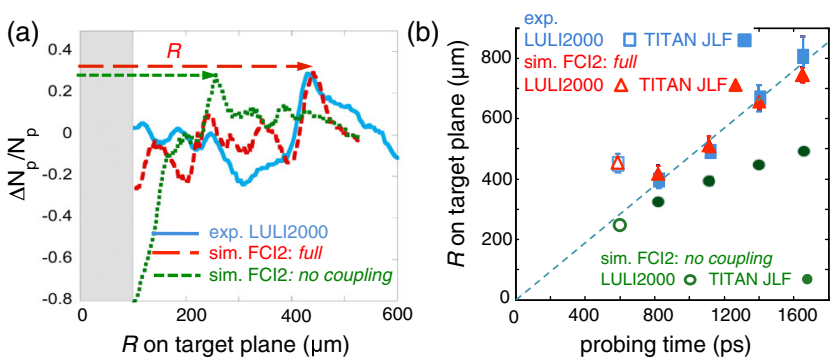

FIG. 2 (color online). (a) Comparison between measured and simulated lineouts of the dose modulation for the LULI2000 experiment. (b) Temporal evolution of $R$ for the two experiments (squares) and in FCI2 simulations for both the "full" (triangles) and "no coupling" (circles) models. Error bars in the simulations are linked to the laser energy uncertainty (evaluated at 5\%).

cases: (1) coupling $B$-field and nonlocal transport with the Nernst and Righi-Leduc effects as described above ("full treatment") or, as a reference case, (2) without these two mechanisms ("no coupling").

With the output of the FCI2 calculations, the proton radiography diagnostic is simulated, using the same conditions (geometry, distances, proton energies) as in the experiments, by calculating the proton trajectories through the simulated field map. The simulated profile of the modulation [see Fig. 2(a)], and notably the radius $(R)$ of the peak of the proton dose accumulation, at the edge of the $B$-field zone, are then compared to the experimental one.

An example of such a calculation, performed in the conditions of the data in Fig. 1(b), is shown in Fig. 2(a) with the experimental dose modulations overlaid. The reference simulated proton deflection pattern (i.e., without the Nernst and Righi-Leduc effects) presents a notable reduced radial extent compared to the experimental one. However, we observe an excellent agreement between experimental results and simulations with FCI2 in the case of the improved heat transport model [21]. Note that such agreement could not be reached when using modeling that did not fully integrate the self-consistent coupling between heat transport and $B$ fields $[15,17]$.

Hence, we can infer that both heat transport and the topology of the $B$ fields are correctly modeled in this case, and that the improved hydrodynamic code is able to model quantitatively the intricate plasma and $B$-field dynamics. The agreement between the experimental data and the simulations, shown in Fig. 2(a) at a particular time during the evolution of the plasma, and for the LULI2000 experiment, is also verified for the TITAN JLF experimental data where it is observed throughout the plasma and $B$-field temporal evolution [see Fig. 2(b)]. Poor agreement is observed when "no coupling" treatment is applied: the field evolution is slower than in the "full treatment case".

Figure 3 presents the $B$-field distributions, in the 2D plane of the simulation, that correspond to the two cases discussed above, as observed at $t=600 \mathrm{ps}$, in the conditions of the LULI2000 experiment. The $B$-field map 
(a)

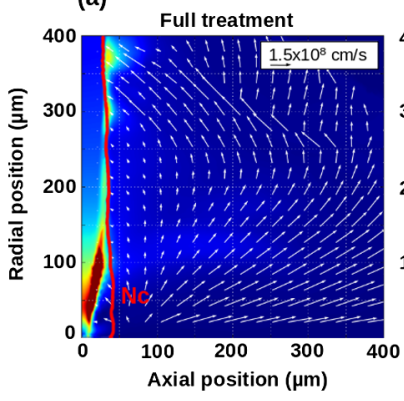

(b)

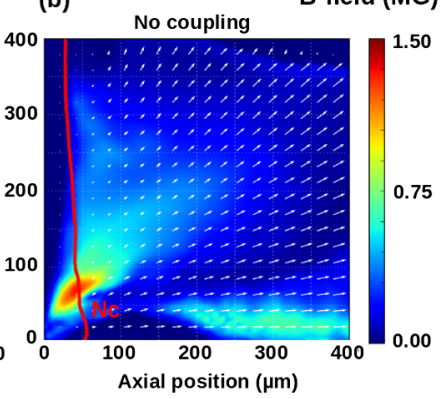

(a)

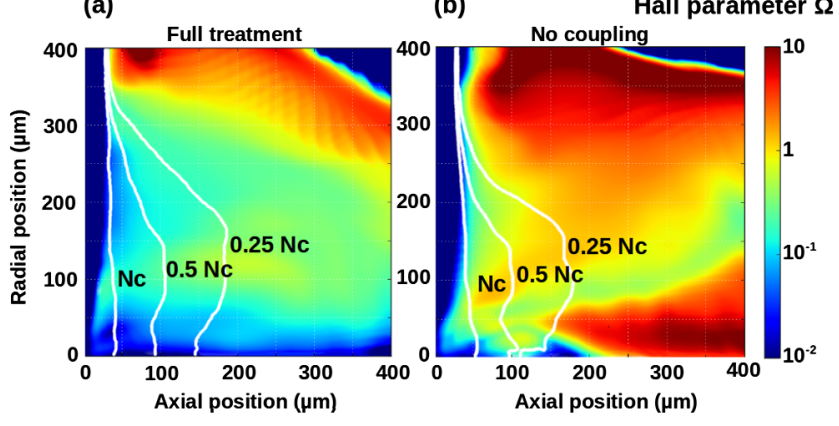

FIG. 3 (color online). 2D FCI2 simulations results at $t=600 \mathrm{ps}$ and performed in the conditions of the LULI2000 experiment. 2D magnetic field spatial distributions (in MG) with isoline of electron density normalized to critical density (corresponding to a $532 \mathrm{~nm}$ laser wavelength). Vector plots of advection velocity $\mathbf{U}=\mathbf{V}_{\text {Flow }}+\mathbf{V}_{\text {Nernst }}$ are also represented. (a) Full treatment. (b) No coupling. Note that due to the axisymmetric geometry, only the upper half plane is simulated.

corresponding to the reference simulation performed without Nernst and Righi-Leduc effects, shown in Fig. 3(b), agrees with the picture derived from previous hydrodynamic modeling (i.e., fields carried by the flow into the underdense part of the plasma) [12-15]. In this case, the amplitude of the $B$ field is strongly reduced and the radial extent of the region exhibiting a strong $B$ field is much smaller (see Fig. 3), compared to the case using the full transport model. This results in a weakly deflected probing proton beam (see Fig. 2, in contradiction with the experimental observation of Fig. 1).

In stark contrast, the $B$-field map for the simulation using the full heat transport model shows how strongly the spatial distribution of the $B$ fields is influenced by the Nernst effect in the case of full treatment: the $B$ fields are advected in the direction of the plasma advection velocity $\mathbf{U}$. Consequently, the direction of convective transport of magnetic fields changes radially and axially between the under dense and over dense plasma regions as witnessed by the advection plot vectors shown in Fig. 3(a).

We observe that, at the center of the target $(<125 \mu \mathrm{m})$ up to the overdense region, the advection is mainly axial. The Nernst advection direction is then opposite to the fluid velocity and is higher in terms of amplitude $\left(V_{\text {Nernst }} \sim 10^{8} \mathrm{~cm} . \mathrm{s}^{-1}\right.$ against $\left.V_{\text {Flow }} \sim 10^{7} \mathrm{~cm} . \mathrm{s}^{-1}\right)$. This leads to a well-known longitudinal convective amplification of the $B$ field, resulting from an increasing electron density associated with a decreasing Nernst velocity $[7,8]$.

In the outer regions (i.e., outside the focal spot size), the same effects lead to a lateral convection of the $B$ field, and to its transport inward [see vector plot from Fig. 3(a)]. As a result, the radial extent of the $B$ field is up to three times the focal spot size, which results in a very large proton deflection (see Fig. 2), consistent with the experimental data (see Fig. 1).

FIG. 4 (color online). 2D FCI2 simulations results at $t=600 \mathrm{ps}$ and performed in the conditions of the LULI2000 experiment. Hall parameter spatial distributions with different isocontour lines of electron density normalized to $532 \mathrm{~nm}$-critical density $\left(n_{c}, 0.5 n_{c}\right.$, and $\left.0.25 n_{c}\right)$. (a) Full treatment. (b) No coupling.

It is interesting to observe how the spatial distribution of the $B$ field shown in Fig. 3 affects the electron magnetization and the nonlocal character of the transport in very different ways, depending on the treatment applied in the simulation. This can be seen by computing the Hall parameter $\Omega$ (the ratio between electron cyclotron frequency and electron-ion mean collision frequency), which allows us to quantify the magnetization of the electrons.

As is shown in Fig. 4, in the case of the simulation run without the Nernst effect, $\Omega$ being higher than 1 in most of the underdense plasma, leads to strong electron magnetization which tends to localize transport and reduces crossfield electron thermal conductivity in a classical way. However, in the case of the full treatment, due to the $B$-field compression toward denser and colder plasma regions [see Fig. 3(a)], the electrons are unmagnetized or weakly magnetized from the center of the target to a radial distance of $350 \mu \mathrm{m}$. This leads to a redelocalization of heat transport, in line with kinetic modeling predictions $[9,10]$. Beyond this position, electrons are strongly magnetized $(\Omega \sim 8)$ reducing the transport of the $B$ field through the overdense plasma.

In summary, we have shown that through a proper treatment of the coupling between $B$ fields and heat transport in a fluid description of the plasma evolution, we can overcome the usual inadequateness of MHD codes and reach a quantitative modeling over long-time scales of the complex plasma evolution following high-power laser irradiation of a solid. We notably observe that the selfgenerated $B$ field is strongly amplified in the overdense plasma while also being radially advected in the critical density region. We stress that this behavior for the plasma and $B$-field evolution is not restricted to the experimental conditions explored in this Letter, but also extends to ICFrelevant conditions: in simulations we performed with the full treatment using the parameters of a NIF-LMJ quadruplet ( $10 \mathrm{~kJ}$ laser energy at a wavelength of $0.35 \mu \mathrm{m}$ ) we 
observe the same type of $B$-field evolution, Based on further simulations we performed, we observe that such an evolution affects the electron transport and, so, the plasma behavior of full ICF experiments in a way that is enough to alter the symmetry necessary to drive the implosion. The results presented here are relative to plastic targets, i.e., relevant for direct-drive ICF. However, we want to point out that we found that the same conclusion regarding the $B$-field topology and heat transport holds true when using high- $Z$ elements $(\mathrm{Au})$, as tested during the TITAN JLF experiment. All these points will be reported in detail in a longer publication [21]. Besides its interest for ICF, we note that the $B$-field topology evidenced here can then be used as relevant initial conditions for reconnection models $[4,5,29,30]$.

We acknowledge the support of the Laboratoire pour l'Utilisation des Lasers Intenses and of the Jupiter Laser Facility technical teams. R. Shepherd and B. Cauble are acknowledged for fruitful discussions. This work was supported by Grant No. E1127 from Région Ile de France, it was partly done within the LABEX Plas@Par project and supported by Grant No. 11-IDEX-0004-02 from Agence Nationale de la Recherche, it was supported by Grant No. 001528 from LaserLab-Europe, by grant agreement 633053 from the EUROfusion consortium, by Discovery Grant No. 26558-2007 from the Natural Sciences and Engineering Research Council of Canada, by the Deutsche Forschungsgemeinschaft Sonderforschungsbereich Transregio 18 and Graduiertenkolleg 1203 programs, by the Engineering and Physical Sciences Research Council Grants No. EP/K022415 and No. EP/I029206/1. This work was supported in part by the Ministry of Education and Science of the Russian Federation under Contract No. 14.Z50.31.0007.

*claude.boniface@cnes.fr julien.fuchs@polytechnique.edu

[1] J. A. Stamper, K. Papadopoulos, R. Sudan, S. Dean, E. McLean, and J. Dawson, Phys. Rev. Lett. 26, 1012 (1971).

[2] M. Yamada, R. Kulsrud, and H. Ji, Rev. Mod. Phys. 82, 603 (2010).
[3] D. Uzdensky, D. A. Uzdensky, Space Sci. Rev. 160, 45 (2011).

[4] R. Smets, N. Aunai, G. Belmont, C. Boniface, and J. Fuchs, Phys. Plasmas 21, 062111 (2014).

[5] A. S. Joglekar, A. G. R. Thomas, W. Fox, and A. Bhattacharjee, Phys. Rev. Lett. 112, 105004 (2014).

[6] L. Spitzer and R. Härm, Phys. Rev., 89, 977 (1953).

[7] A. Nishiguchi, T. Yabe, M. G. Haines, M. Psimopoulos, and H. Takewaki, Phys. Rev. Lett. 53, 262 (1984).

[8] T. H. Kho and M. G. Haines, Phys. Rev. Lett. 55, 825 (1985).

[9] J. F. Luciani, P. Mora, and A. Bendib, Phys. Rev. Lett. 55, 2421 (1985).

[10] C. P. Ridgers, R. J. Kingham, and A. G. R. Thomas, Phys. Rev. Lett. 100, 075003 (2008).

[11] S. Atzeni and J. Meyer-Ter-Vehn, The Physics of Inertial Fusion (Oxford University Press, Oxford, England, 2004).

[12] C. K. Li et al., Phys. Rev. Lett. 97, 135003 (2006).

[13] C. K. Li et al., Phys. Rev. Lett. 99, 015001 (2007).

[14] C. K. Li et al., Phys. Plasmas 16, 056304 (2009).

[15] L. Lancia, Ph.D. Thesis, École Polytechnique and Università di Roma "La Sapienza," 2010.

[16] M. J-E. Manuel et al., Phys. Plasmas 20, 056301 (2013).

[17] L. Lancia et al., Laser Part. Beams 31, 653 (2013).

[18] S. I. Braginskii, in Review of Plasma Physics, edited by M. A. Leontovitch (Consultants Bureau, New York, 1965), Vol. 1, pp. 205-311.

[19] R. D. Petrasso et al., Phys. Rev. Lett. 103, 085001 (2009).

[20] L. Willingale et al., Phys. Rev. Lett. 105, 095001 (2010).

[21] C. Boniface et al. (to be published).

[22] R. D. Snavely et al., Phys. Rev. Lett. 85, 2945 (2000).

[23] C. A. Cecchetti et al., Phys. Plasmas 16, 043102 (2009).

[24] T. E. Cowan et al., Phys. Rev. Lett. 92, 204801 (2004).

[25] E. Dattolo et al., Phys. Plasmas 8, 260 (2001).

[26] P. Nicolai, M. Vandenboomgaerde, B. Canaud, and F. Chaigneau, Phys. Plasmas 7, 4250 (2000); Ph. D. Nicolaï, J.-L. A. Feugeas, G. P. Schurtz. Phys. Plasmas 13, 032701 (2006).

[27] L. Biermann, Z. Naturforsch. 5a, 65 (1950).

[28] G. Schurtz, Ph. D, Nicolai, and M. Busquet, Phys. Plasmas 7, 4238 (2000).

[29] P. M. Nilson et al., Phys. Rev. Lett. 97, 255001 (2006)

[30] W. Fox, A. Bhattacharjee, and K. Germaschewski, Phys. Rev. Lett. 106, 215003 (2011); W. Fox, A. Bhattacharjee, and K. Germaschewski, Phys. Plasmas 19, 056309 (2012). 